\title{
AVALIAÇÃO DE TECNOLOGIA EM SAÚDE. II. A análise de custo-efetividade
}

\author{
Silvia Regina SECOLI, Marcelo Eidi NITA, \\ Suzane Kioko ONO-NITA and Moacyr NOBRE
}

\begin{abstract}
RESUMO - O advento de novas tecnologias na saúde causou impacto nos indicadores clínicos e econômicos. Os métodos de pesquisa que incorporam conceitos da economia da saúde e epidemiologia clínica permitem avaliar a eficiência de novas tecnologias, por exemplo, através da análise de custo-efetividade. Este é um instrumento de análise de valor das intervenções em saúde. A metodologia, análise de custo-efetividade, é condição determinante da moderna prática de cuidados à saúde, pois as opções terapêuticas hoje disponíveis no Sistema Único de Saúde (SUS) ou no sistema de saúde suplementar do Brasil passam necessariamente por tal análise, logo o sistema de saúde bem como os profissionais da saúde são levados a reexaminar os benefícios e custos de suas ações para assegurar que haja incorporação das tecnologias mais eficientes. Neste segundo artigo sobre avaliação de tecnologia em saúde reviram-se os conceitos de análise de custo-efetividade, os passos envolvidos na sua execução e o método para a análise crítica dos resultados.

DESCRITORES - Avaliação de programas e projetos de saúde. Análise custo-eficiência. Revisão da utilização de recursos de saúde. Sistemas de saúde.
\end{abstract}

\section{INTRODUÇÃO}

O advento de novas tecnologias de saúde como fármacos (p.ex. imunobiológicos), equipamentos médicos (p.ex. grampeadores para cirurgia), exames diagnósticos, entre outros, causaram impacto nos indicadores clínicos e econômicos. Nesse cenário, a importância de estudos que conjuguem estes elementos provém não de justificativas acadêmicas ou políticas, mas sobretudo, da constatação de que os gastos com saúde vêm crescendo em ritmo acelerado, podendo afetar a sustentabilidade dos sistemas de saúde ${ }^{(17)}$.

Em âmbito mundial, os sistemas de saúde, na tentativa de melhorar a eficiência, a expansão da oferta e cobertura, e incorporar novas tecnologias que sejam comprovadamente eficazes e seguras, vêm adotando estratégias de monitoramento e avaliação da assistência, utilizando-se de instrumentos oriundos da economia da saúde e da epidemiologia clinica ${ }^{(15)}$.

Considerando o jargão técnico da área, a eficácia considera o benefício no contexto artificial dos experimentos clínicos, a efetividade diz respeito ao benefício observado no mundo real do dia-a-dia do indivíduo, enquanto a eficiência se refere ao benefício ponderado pelo custo frente às opções terapêuticas existentes.

A eficiência avaliada pelo estudo de custo-efetividade é um instrumento de análise de valor das intervenções em saúde. $\mathrm{O}$ método busca preencher a lacuna existente entre as preferências (subjetividade) e a ciência (objetividade, validade, reprodutibilidade). Nesse contexto, o conceito de "valor" deve ser compreendido numa concepção ampla, referindo-se às preferências que um indivíduo ou sociedade apresentam face a escolhas mutuamente excludentes, não apenas a quantia em dinheiro trocada na aquisição de bens ou serviços. Traduzir valor para uma dimensão apenas financeira não constitui bom caminho para as sociedades e muito menos para as ações de saúde. Oscar Wilde, no século XIV já mencionava: "O que é um cínico? Um homem que conhece o preço de qualquer coisa, mas não sabe o valor de nada". Em contrapartida, não se pode deixar de considerar que o custo das novas tecnologias tem que ser gerenciado, sob o risco de penalizar o próprio segmento e outras áreas de interesse social ${ }^{(8)}$.

A seleção de uma opção terapêutica é problema cientifico e deve guiar-se pelo estado de arte e nível de conhecimento profissional, embora na tomada da decisão de utilização do uso de uma tecnologia estejam envolvidos aspectos políticos, sociais, éticos e mesmo culturais. E, hoje, os aspectos econômicos são absolutamente fundamentais. Ademais, à medida que a demanda no Sistema Único de Saúde (SUS) aumenta e os recursos tornam-se cada vez mais escassos, o próprio sistema de saúde, bem como os profissionais da saúde têm de reexaminar os benefícios e custos de suas ações para assegurar que haja uma implementação efetiva das intervenções ${ }^{(8)}$.

Tendo em vista a importância de investigações acerca do "valor" das intervenções de saúde, da

Departamento de Enfermagem Médico-Cirúrgica, Escola de Enfermagem, Universidade de São Paulo (USP); ${ }^{2}$ Departamento de Gastroenterologia, Faculdade de Medicina, USP; ${ }^{3}$ Departamento de Epidemiologia Clínica, Instituto do Coração (INCOR), Hospital das Clinicas, Faculdade de Medicina, USP, São Paulo, SP. Correspondência: Dr. Marcelo Eidi Nita - Av. Dr. Eneas de Carvalho Aguiar, 255 - $9^{\circ}$ andar - 05403-000 - São Paulo, SP. E-mail: menita@usp.br 
necessidade cada mais explicita da tomada de decisão ser pautado em eficiência, o propósito do presente artigo é abordar os princípios que regem os estudos de custo-efetividade e destacar as etapas necessárias à elaboração destes. A disseminação desta metodologia é condição determinante da moderna prática de cuidados à saúde, pois por Lei as opções terapêuticas hoje disponíveis no SUS ou no sistema de saúde suplementar, passam por tal avaliação de custo e efetividade, assim, a prática de cuidados à saúde acaba sendo influenciada por esta análise.

\section{ANALISE DE CUSTO-EFETIVIDADE - ACE}

O conceito custo-efetividade na saúde surgiu em países desenvolvidos, no final dos anos de $1970^{(17)}$. A ACE é uma metodologia de síntese em que os custos são confrontados com desfechos clínicos. O objetivo da ACE é avaliar o impacto de distintas alternativas, que visem identificá-las com melhores efeitos do tratamento, geralmente, em troca de menor custo. Portanto, uma característica importante é que os estudos de ACE são sempre comparativos e explícitos e se destinam a selecionar a melhor opção para atingir a eficiência.

Nesse tipo de análise, os custos são medidos em unidades monetárias e os desfechos em unidades clínicas, tais como mortalidade ou hospitalizações evitadas - uma vantagem importante, tendo em vista que esses desfechos, frequentemente, são de uso corrente pelos clínicos ${ }^{(2,17)}$. Os resultados da ACE são expressos por um quociente, em que o numerador é o custo e o denominador a efetividade (custo/efetividade). Desse modo, a ACE é expressa em termos do custo por unidade clínicas de sucesso. Por exemplo, custo por anos de vida ganhos, ou por mortes evitadas, ou por dias sem dor, ou por ausência de complicação, ou ainda por hospitalizações evitadas.

$\mathrm{Na} \mathrm{ACE}$ as alternativas comparáveis podem ser colocadas em um plano de custo-efetividade. Trata-se de uma representação gráfica, cujo ponto do plano em que os eixos x e y se cruzam indica o ponto de origem dos custos e efetividade para o fator padrão de comparação (Figura 1). Este resultado gráfico representado na Figura 1 facilita a identificação da alternativa custo-efetiva.

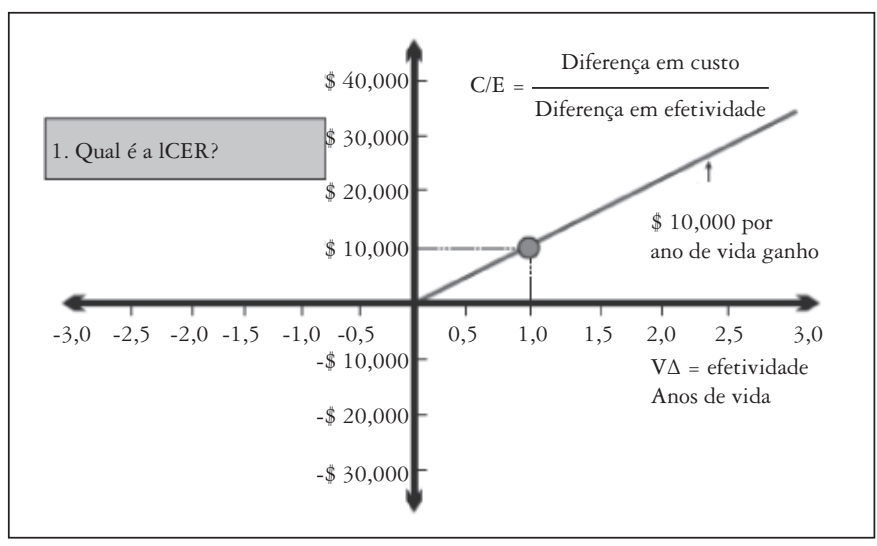

FIGURA 1. Gráfico de custo-efetividade de distintas alternativas terapêuticas ${ }^{(1)}$
Nas discussões sobre os resultados da ACE, frequentemente, entretanto, há equívocos conceituais que interferem na interpretação dos resultados. O termo, muitas vezes, é usado como sinônimo de mais barato, independente da eficácia das alternativas comparadas; ou sinônimo de mais eficaz, independente dos custos; ou aplica-se conceito custo-efetivo para situações em que a eficácia das opções foi semelhante. Todavia, a aplicação correta do termo destina-se a situações em que os benefícios extras de uma opção terapêutica compensam o custo adicional ao comparador ${ }^{(17)}$.

A finalidade das análises de ACE é identificar a alternativa custo-efetiva. Basicamente, há duas maneiras de afirmar que uma intervenção é custo-efetiva - o processo comparativo das alternativas e o uso de valores-limite (threshold). A Figura 2 ilustra exemplo desse conceito.

\begin{tabular}{|lccc|}
\hline Alternativas & Custo $(\mathbf{R} \$)$ & $\begin{array}{c}\text { Efetividade } \\
\text { (dias sem sintomas) }\end{array}$ & $\begin{array}{c}\text { Relação custo-efetividade } \\
\text { incremental }\end{array}$ \\
\hline Terapia A & 2.000 & 5 & $\mathrm{R} \$ 400,00 /$ dia sem sintoma \\
Terapia B & 6.000 & 3 & $\mathrm{R} \$ 2.000,00 /$ dia sem sintoma \\
Terapia C & 4.800 & 6 & $\mathrm{R} \$ 800,00 /$ dia sem sintoma \\
Terapia D & 3.000 & 4 & $\mathrm{R} \$ 750,00 /$ dia sem sintoma \\
\hline
\end{tabular}

FIGURA 2. Comparações de custo-efetividade para terapias medicamentosas hipotéticas

A terapia A é custo-efetiva em relação às demais intervenções, pois exige menor gasto para obter 1 dia sem sintoma (ela pode ser chamada de alternativa dominante); a terapia B é a menos desejável pois tem a pior relação custo-efetividade incremental (ela pode ser chamada de alternativa dominada), pois implica em maior gasto para obter o desfecho clínico, ou seja, 1 dia sem sintoma. As terapias C e D são custo-efetivas em relação a $\mathrm{B}$, mas não são custo-efetivas em relação a $\mathrm{A}$.

A partir desse exemplo, dentro de um contexto de baixa complexidade e sem limitação orçamentária, os resultados apontariam para a escolha da terapia $\mathrm{A}$, com a melhor relação de custo-efetividade entre todos. Não haveria nenhuma discussão em relação à intervenção $\mathrm{B}$, cujo custo é muito elevado e o benefício clínico é o menor da série. Todavia, qual seria a interpretação das demais? Por exemplo, para fins didáticos de discussão, a observação dos valores de custo e de efetividade, isoladamente, revela que no caso da terapia D é possível poupar dinheiro, embora perdendo efetividade.

De modo geral, a análise deve ser elaborada tendo como referência uma alternativa que sirva como padrão. Na prática é desejável que o padrão represente um protocolo vigente ou um fármaco padronizado, muito embora seja possível realizar análises usando outros comparadores (de forma direta ou indireta), possivelmente acarretando prejuízos ao significado de valor comparativo entre a inovação e o padrão corrente ${ }^{(8)}$.

A análise de custo consequência (ACC) é uma variante da ACE. Na ACC, as consequências (ou benefícios clínicos) são apresentadas de forma itemizada e separada dos custos. Isto é, ACC é similar a ACE nos desfechos escolhidos. Todavia, difere da ACE, pois os inúmeros desfechos são apresentados de forma desagregada, ao invés de focar em simples desfecho. 
A vantagem da ACC é a possibilidade de indicar os resultados mais relevantes e o custo associado a ela, facilitando o processo de interpretação $0^{(4,13)}$. A ACC e ACE devem ser vistas como complementares, permitindo, ao analisar por itens, identificar quais resultados têm o maior peso no resultado da razão ou relação custo-efetividade incremental.

\section{ETAPAS DA CONSTRUÇÃO DA ACE}

A construção de estudos dessa natureza encontra-se alicerçada em procedimentos básicos usados na execução de avaliações econômicas e cuja qualidade encontra-se atrelada ao seguimento parcial ou total dessas etapas ${ }^{(17)}$

O ponto inicial e um dos principais da ACE é a definição objetiva do problema a ser investigado, ou seja, qual a pergunta da pesquisa. A formulação equivocada ou a organização pouco estruturada da pergunta, certamente, dificultará o delineamento do estudo e poderá comprometer os resultados da analise.

Para melhor entender esse passo, considerou-se o seguinte caso clínico: Qual é a alternativa terapêutica mais custoefetiva na erradicação da dispepsia não-ulcerosa associada com Helicobacter pylori (HP)?

A seleção das alternativas terapêuticas ou tecnologias em saúde (por exemplo: medicamento, equipamento, procedimento endoscópico ou serviço) a serem estudadas é ponto crítico e deve ser baseada em uma revisão sistemática da literatura adaptada para a perspectiva da análise. Na pergunta proposta as alternativas se referem aos medicamentos utilizados para a erradicação do $H P$.

A seguir, é importante a definição da perspectiva da análise (ponto de vista), que consiste em indicar quem é o interessado na resposta do estudo. A perspectiva interfere na seleção das categorias de custos e dos desfechos a serem avaliados. Por exemplo, na perspectiva de um sistema de saúde (p. ex. SUS, sistema de saúde suplementar ou sistema de saúde local), frequentemente, são selecionados custos diretos médicos e os desfechos associados à recuperação do paciente, visto que são indicadores de impacto administrativo-financeiro. $\mathrm{Na}$ ótica da sociedade, custos indiretos como, por exemplo, absenteísmo associado à doença, bem como o desfecho de qualidade de vida, são importantes para medir o impacto da intervenção e, por isso, selecionados na análise.

O próximo passo é a seleção dos desfechos, que são estratificados em finais (DF) e intermediários (DI). Os DF são os de maior impacto para o paciente ou para a sociedade, porém podem levar vários anos para ocorrer, demandando estudos com grandes amostras e longo tempo de seguimento; morte ou infarto são exemplos de $\mathrm{DF}^{(7)}$. Um DF considerado padrão ouro é o quality-adjusted life years (QALY) ou anos de vida ajustados pela qualidade (AVAQ), que permite expressar o ganho mais desejável pelo paciente, ou seja, números de anos vividos ajustados pela qualidade. Os DI são mais comumente usados nos estudos com o intuito de reduzir o tempo, a complexidade e os custos. Entretanto, se não refletem o benefício da intervenção a longo prazo, seus resultados podem ser questionáveis. São exemplos desse tipo de desfecho exames laboratoriais (redução de colesterol ou da glicemia) ou exames de imagens (tal como, ultrassonografia de nódulos hepáticos). Para um DI ser substituto eficaz do DF, o produto testado deve agir e alterar o DI e também causar alteração proporcional no $\mathrm{DF}^{(6)}$. Desse modo, nem sempre os desfechos selecionados pelos pesquisadores ou clínicos retratam o benefício real da intervenção de saúde e, por isto, devem ser criticamente avaliados. No caso clínico do HP citado, os desfechos podem ser avaliados por resolução ou alivio dos sintomas da dispepsia, que pode ser mensurada em dias ou semanas.

Os desfechos podem, também, ser apresentados por meio do Number Needed to Treat ou Número Necessário para Tratar (NNT), medidas talvez mais práticas, que permitem avaliar o benefício de uma dada terapia em detrimento de outra $^{(10)}$. O NNT representa o número de indivíduos que devem ser tratados para obter um único desfecho favorável, em comparação a outra alternativa terapêutica. Apesar de tratarse de um indicador epidemiológico, ele oferece informações numéricas de pesquisas que, aos olhos do clínico, têm mais sentido, inclusive para o paciente. A obtenção dos desfechos (DF ou DI) pode derivar-se de diversas fontes como revisão sistemática, ensaios clínicos controlados e randomizados, estudos epidemiológicos, base de dados, registros em prontuários, opiniões de especialistas, podendo a coleta de informações ocorrer de modo prospectivo ou retrospectivo. Atualmente considera-se que os estudos mais apropriados para fornecer informações acerca de desfechos são a revisão sistemática e a metanálise.

A próxima etapa é a seleção das categorias de custos, quais sejam: custos diretos e indiretos. O cálculo do custo de uma intervenção, independente da fonte utilizada, raramente é tarefa simples e se torna mais complexa na medida em que se busca aproximar o modelo analítico adotado ao que acontece no mundo real. Por exemplo, para um paciente com hemorragia gástrica, o modelo compreenderia apurar todos os procedimentos e recursos consumidos (medicamentos, materiais, exames, diárias, honorários profissionais, entre outros) de certo número de indivíduos, e determinar o custo por média aritmética simples. No entanto, a observação do mundo real deve mostrar que o fluxo de eventos não é tão harmônico quanto se desejaria, pois ocorrem casos em que há ineficácia terapêutica, reações adversas, mudanças de conduta, externalidades, efeito de variáveis (idade, estado geral do paciente) que podem ocorrer com regularidade ${ }^{(8)}$. $\mathrm{Na}$ medida em que estas interferências geram impacto nos custos e/ou desfechos, torna-se necessário que as mesmas sejam incluídas nos cálculos da ACE, com a devida ponderação entre a frequência de ocorrência e a magnitude de seus efeitos.

A elaboração desses cálculos (custos e benefícios) é feita por meio de modelos de decisão clínica e avaliação econômica como, o mais simples, a árvore de decisão, até os mais complexos como os modelos de Markov, simulações de Monte Carlo, métodos bayesianos e outros ${ }^{(2,8,11)}$. Os modelos têm de ter uma estrutura representativa da análise e devem estar calcados, inicialmente, em conhecimento da história natural da doença 
e do curso das alternativas terapêuticas, ou seja, considerar horizonte de tempo da análise, de maneira adequada. Não considerar esse aspecto pode incorrer no desenvolvimento de um modelo que não reflete a epidemiologia e a história natural da doença e das alternativas estudadas. Enfermidades crônicas, como doença de Crohn ou câncer, devem ter um horizonte por toda a vida, enquanto situações clínicas como sintomas de dispepsia ou o tratamento do HP podem ser adequadamente representadas por desfechos em prazo de, por exemplo, 1 ano de seguimento. Os modelos podem, também, ser desenvolvidos em programas comerciais (por exemplo: TreeAge $\left.{ }^{\circledR}\right)$, ou em programas genéricos como o MS Excel (Microsoft, US).

Outro aspecto a ser considerado no cálculo do custo e do benefício, é a taxa de desconto. A realização da análise, geralmente, ocorre em momento temporal distinto da tomada de decisão acerca dos resultados apontados nesta. Para comparar os custos e desfechos em diferentes momentos no período de tempo, utiliza-se a taxa de desconto, que segundo alguns autores, deve-se situar entre 3\%-5\% ao ano ${ }^{(5,9)}$. Trata-se da aplicação de taxa para obter o valor presente dos custos que ocorrem nos distintos tempos.

Os resultados fornecidos pela ACE podem ser apresentados como razão custo-efetividade incremental (RCEI) ou "Incremental Cost Effectiveness Ratio" (ICER) e validados através de análise de sensibilidade.

A RCEI é a diferença nos custos entre duas alternativas que estamos estudando, dividida pela diferença da efetividade das alternativas terapêuticas. Assim, o cálculo é realizado por meio da seguinte fórmula:

[Custo da intervenção X] - [Custo da intervenção Y]

[Efetividade da intervenção X] - [Efetividade da intervenção Y]

Os resultados representam, então, o custo incremental ou adicional por benefício incremental ou adicional obtido. Por exemplo, como custo por anos de vida ganho ou custo por complicação evitada ou, ainda, custo por QALY.

A análise de sensibilidade é uma técnica de avaliação da incerteza, acerca de toda e qualquer covariável contemplada no modelo econômico, ou seja, a respeito de custos ou de desfechos. Ela permite comprovar o grau de estabilidade ou robustez dos resultados e até que ponto o resultado da RCEI é mantido, quando valores das variáveis principais são modificados dentro de limites plausíveis ${ }^{(12)}$. Elas podem ser realizadas variando uma (univariada) ou várias covariáveis simultaneamente. A técnica de caso referência, com o melhor e o pior cenário, é muito utilizada para se estudar uma faixa de resultados. A análise de sensibilidade probabilística é uma técnica mais elaborada e permite estudar todas as variáveis simultaneamente. Para muitos autores é a técnica mais adequada e permite indicar a probabilidade de o resultado ser custo efetivo, deixando ao pesquisador a decisão de considerar os resultados custo efetivos ou não ${ }^{(12,16)}$.

Os estudos de ACE têm sido construídos para comparar: alternativas terapêuticas no tratamento da hepatite crônica
$\mathrm{B}^{(3)}$; programas de saúde, por exemplo $\mathrm{ACE}$ da orientação nutricional em pacientes com obesidade, ou intervenções, por exemplo ACE do uso ligadura elástica endoscópica em pacientes com varizes de esôfago; medicamentos, por exemplo ACE da utilização de medicamentos biológicos em doença de Crohn; produtos, por exemplo ACE do uso de grampeadores em cirurgias, entre outros.

\section{Estudo de caso clínico}

Moayyedi et al. ${ }^{(14)}$ avaliaram a eficácia, através de uma revisão sistemática da literatura, e o custo-efetividade, através de uma avaliação econômica, da erradicação do Helicobacter pylori (HP) em pacientes com dispepsia não-ulcerosa. Tipicamente, pois, a análise se inicia com uma revisão da literatura, utilizando as técnicas da medicina baseada em evidência. Neste caso, os autores encontraram 12 artigos com critérios adequados para serem analisados, num total de 2.541 pacientes. $O$ resultado indicou que a erradicação foi superior ao placebo no tratamento da dispepsia não-ulcerosa. A avaliação econômica foi realizada através de um modelo de Markov, empregando um programa comercial denominado, Tree Age versão 3.5 (Tree Age software, Willianstown, MA, USA). Os dados de redução do risco relativo para os sintomas dispépticos obtidos na revisão sistemática foram utilizados como parâmetro de efetividade para "popular" o modelo de Markov. A perspectiva foi a de um serviço de saúde, incorporando os custos dos medicamentos e a visita a um médico generalista. Os resultados foram demonstrados como custo por mês livre de dispepsia. $O$ custo da erradicação do HP foi de \$56 por mês livre de dispepsia durante o primeiro ano de tratamento, resultado que deve ser custo efetivo. As incertezas do modelo estudado foram testados para sua robustez através de análise de sensibilidade univariada e probabilística, usando simulação de Monte Carlo.

Este caso exemplifica uma avaliação de custo efetividade e os principais resultados oriundos de sua execução. Assim, os autores inicialmente avaliam se a terapia de erradicação de HP, a tecnologia sendo discutida, é eficaz através de revisão sistemática da literatura. Comprovada que a tecnologia funciona, então, os autores passaram para a próxima fase que é a da avaliação do custo-efetividade, através de um modelo de Markov. Os resultados indicam que esta é uma estratégia, quando comparada com placebo, custo-efetiva. Assim, os profissionais da saúde têm em dois critérios, a eficácia e a eficiência, a constatação de uma tecnologia que pode ser considerada para a efetiva implementação na prática do dia-a-dia.

\section{CONCLUSÃO}

A avaliação de tecnologia em saúde, por meio do uso da ACE é o método mais indicado para a avaliação de duas ou mais alternativas terapêuticas, procedimentos ou serviços de saúde. Ela permite a análise combinada de benefícios clínicos e os custos associados, e fornece dados objetivos e explícitos para decidir a opção mais eficiente. Esse tipo de análise representa instrumento auxiliar no processo decisório de escolhas de novas tecnologias em saúde. Porém, aspectos 
de ordem cultural e epidemiológica que intervêm na decisão devem acompanhá-la. Na prática clínica, muitas vezes, uma alternativa terapêutica pode ser tecnicamente viável e economicamente satisfatória, quando comparada a outras, mas culturalmente indesejável; neste caso, dificilmente será adotada. A realização disseminada desse tipo de análise, seguramente permitirá a seleção de opções terapêuticas que efetivamente funcionam, com a exclusão daquelas com benefícios duvidosos e, quem sabe, o desenvolvimento de sistemas de saúde economicamente sustentáveis neste ambiente de custos crescentes no SUS e sistema de saúde privado do Brasil.

Secoli SR, Nita ME, Ono-Nita SK, Nobre M. Health technology assessment. II. Cost effectiveness analysis. Arq Gastroenterol. 2010;47(4):329-33.

ABSTRACT - New health technologies have made an impact in clinical and economic outcomes. Therefore, research methodologies that allow to evaluate the efficiency of these new technologies such as cost-effectiveness analysis are necessary. Cost-effectiveness analysis assess the value of health care interventions or drugs, the technology. Cost-effectiveness analysis is also deemed a determinant of modern health care practice, because the therapeutic options available at public (SUS) or private health care system must go through a formal health technology assessment in Brazil; thus, both the health care system and the health care professionals have to reevaluate the clinical consequences and costs of their actions to assure that the most efficient technologies are the one used in the practice. In this second article about health technology assessment we review the concepts of cost-effectiveness analysis, the steps involved in performing such analysis, and the criteria most frequently used to critically review the results.

HEADINGS - Program evaluation. Cost efficiency analysis. Utilization review. Health systems.

\section{REFERÊNCIAS}

1. Augustovski F, Andres Pichon-Riviere A, Rubinstein A. Critérios utilizados pelos sistemas de saúde para a incorporação de tecnologias. In: Nita ME, Campino ACC, Secoli SR, Sarti FM, Nobre M, Costa AM, Ono-Nita SK, Carrilho FJ, editores. Avaliação de tecnologias em saúde. Porto Alegre: Artmed; 2010. p.493-505.

2. Brasil. Ministério da Saúde. Avaliação econômica em saúde: desafios para gestão no Sistema Único de Saúde. Brasília: Editora do Ministério da Saúde, 2008.

3. Costa AM, L'Italien G, Nita ME, Araujo ES. Cost-effectiveness of entecavir versus lamivudine for the suppression of viral replication in chronic hepatitis B patients in Brazil. Braz J Infect Dis. 2008;12:368-73.

4. Detsky AS, Laupacis A. Relevance of cost-effectiveness analysis to clinicians and policy makers. JAMA. 2007;298:221-4

5. Drummond M, McGuire A. Economic evaluation in health care: merging theory with practice. New York: Oxford University Press; 2001. p.236-55.

6. Ellenberg S, Hamilton JM. Surrogate endpoints in clinical trials: cancer. Stat Med. 1989;8:405-13.

7. Fleming TR, DeMets DL. Surrogate end points in clinical trials: are we being misted. Ann Intern Med. 1996;125: 605-13.

8. Follador W, Secoli SR. A farmacoeconomia na visão dos profissionais da saúde In: Nita ME, Campino ACC, Secoli SR, Sarti FM, Nobre M, Costa AM, OnoNita SK e Carrilho FJ. Avaliação de tecnologias em saúde. Porto Alegre: Artmed; 2010. p.248-68.
9. Gold MR, Siegel JE, Russell LB, weinstein mc. Cost-effectiveness in health and medicine. New York: Oxford University Press; 1996. p.214-38.

10. Hildebrandt M, Vervölgyi E, Bender R. Calculation of NNTs in RCTs with timeto-event outcomes: a literature review. BMC Med Res Methodol. 2009;20:9-21.

11. International Society for Pharmacoeconomics and Outcomes Research - ISPOR. Modelling Best Practices. Disponivel em http://www.ispor.org [ acesso em 2 Mai 2009].

12. Manning WG, Fryback DG, Weinstein MC. Reflecting uncertainty in costeffectiveness analysis. In: Gold MR, Siegel JE, Russel LB, Weinstein MC. Costeffectiveness analysis in health and medicine. New York: Oxford University Press; 1996. p.247-75.

13. Mauskopf JA, Paul JE, Grant DM, Stergachis A. The role of cost-consequence analysis in healthcare decision-making. Pharmacoeconomics. 1998;13:277-88.

14. Moayyedi P, Soo S, Deeks J, Forman D, Mason J, Innes M, Delaney B. Systematic review and economic evaluation of Helicobacter pylori eradication treatment for non-ulcer dyspepsia. Dyspepsia review group. BMJ. 2000;321:659-64.

15. Nita ME, Secoli SR, Nobre M, Ono-Nita SK. Métodos de pesquisa em avaliação de tecnologia em saúde. Arq Gastroenterol. 2009;46:252-5.

16. Nita ME, Secoli S, Sarti FM. Analise de sensibilidade. In: Nita ME, Campino ACC, Secoli SR, Sarti FM, Nobre M, Costa AM, Ono-Nita SK, Carrilho FJ. Avaliação de tecnologias em saúde. Porto Alegre: Artmed; 2010. p.368.

17. Secoli SR, Padilha KG, Litvoc J, Maeda ST. Farmacoeconomia : perspectiva emergente no processo de tomada de decisão. Ciên Saúde Coletiva. 2005;10:28796.

\section{LEITURA RECOMENDADA}

1. Inadomi J.M. Decision analysis and economic modelling: a primer. Eur $\mathbf{J}$ Gastroenterol Hepatol. 2004:16:535-42.

2. Nita ME, Campino ACC, Secoli SR, Sarti FM, Nobre M, Costa AM, Ono-Nita SK, Carrilho FJ. Avaliação de tecnologias em saúde. Porto Alegre: Artmed; 2010. p.1-600

3. Nobre M, Bernardo W. Prática clínica baseada em evidência. Rio de Janeiro: Elsevier; 2007. p.1-238.
4. Petitti, DB. Meta-analysis, decision analysis and cost-effectiveness analysis. 2nd ed. Oxford University Press;2000.

5. Rubenstein JH, Inadomi JM. Evidence based medicine (EBM) in practice: applying results of cost-effectiveness analysis. Am J Gastroenterol. 2006;101: 1169-71.

6. Sonnenberg A. Decision analysis in clinical gastroenterology. Am J Gastroenterol. 2004;99:163-9.

7. Uyl-de Groot CA. Economic evaluation of cancer therapies: more and better studies will lead to better choices in cancer care. Eur J Cancer. 2006;42: 2862-6 